\title{
Cladribine an Alternative for Midostaurine in FLT3 Positive
}

\section{Acute Myeloid Leukemia}

\author{
Marlies EHM Van Hoef* \\ Department of Medical Affairs, Lipomed AG, Switzerland
}

*Corresponding author: Marlies EHM Van Hoef, Department of Medical Affairs, Lipomed AG, Fabrikmattenweg 2/4, 4114 Arlesheim, Switzerland, Tel: 0041-617159651; Email: vanhoef@vanhoefconsulting.com

Keywords: Cladribine; Midostaurine; Sorafenib; FLT3 positive acute myeloid leukemia

\section{Letter to Editor}

Volume 2 Issue 2

Received Date: October 17, 2018

Published Date: October 26, 2018

DOI: $10.23880 /$ hij-16000134

\section{Letter to the Editor}

Midostaurin is registered for the indication newly diagnosed acute myeloid leukemia that is FLT3 mutation positive in combination with standard cytarabine and daunorubicine induction and cytarabine consolidation. The registration is based on a study of 717 patients that examined the addition of midostaurin $50 \mathrm{mg}$ orally twice daily or placebo to standard induction on days 8 to 21 of a cycle for a maximum of two induction cycles [1]. In case of complete remission patients received four cycles of 28 days cytarabine consolidation with midostaurin $50 \mathrm{mg}$ twice daily on days 8 to 21 . Patients who remained in complete remission after consolidation therapy entered a maintenance phase in which patients received midostaurin $50 \mathrm{mg}$ twice daily or placebo for twelve 28 days cycles. The complete remission rate was $58.9 \%$ versus $53.5 \%$ respectively. Median disease free survival was $26.7 \mathrm{~m}$ versus 15.5 months respectively. The 4-years overall survival was $51.4 \%$ and $44.3 \%$ respectively. The median overall survival was 74.4 months versus 25.6 months respectively. This difference was explained by the inflection rates of the Kaplan Meyer curves. The hazard ratio for death 0.78 (95\% CI $0.63-0.96)$ reflected the magnitude of the benefit.

Zhang, et al. [2] reported the addition of sorafenib to standard chemotherapy. Details do not exist as the publication is in Chinese. The study included 55 patients with a FLT3 mutation. The complete response rate in the chemotherapy plus sorafenib group was $86.4 \%$ and in the chemotherapy plus placebo group $35.5 \%$. The one year progression free survival was $75.9 \%$ versus $42.4 \%$ respectively and the one year overall survival $78.3 \%$ vs $50 \%$ respectively.

A study in elderly with sorafenib in combination with intensive chemotherapy did not result in significant improvement in event free and overall survival in the FLT3 mutation positive subgroup [3]. The patients had received standard induction chemotherapy plus sorafenib $400 \mathrm{mg}$ twice daily or placebo from three days post induction to three days prior to next induction chemotherapy course. Patients with complete remission had received two cycles consolidation cytarabine and the same sorafenib or placebo regimen. Patients in complete remission received maintenance with sorafenib or placebo. This regimen is more or less comparable with that in the study with midostaurin, but did not yield positive results.

Libura, et al. [4] reported a retrospective study of 227 patients in which 55 patients were identified that were FLT3-ITD positive. In the original study standard induction chemotherapy for maximal two cycles with daunorubicine and cytabine and cladribine $5 \mathrm{mg} / \mathrm{m}^{2}$ days 1-5 (DAC) versus placebo (DA) were compared $[5,6]$. Consolidation treatment had consisted of two courses of cytarabine day 1 to 3 , mitoxantrone days 3 to 5 and high dose cytarabine twice daily days 1,3 and 5, in the DAC arm accompanied by cladribine $5 \mathrm{mg} / \mathrm{kg}$ i.v. on days 1,3 and 5 . Maintenance treatment was given for up to two years and consisted of daunorubicine i.v. day 1 and cytarabines twice daily days 1 to 5 , alternated with 6 thioguanine days 
1 to 5 and cytarabine twice daily days 1 to 5 . Patients in the DAC arm received cytarabine $5 \mathrm{mg} / \mathrm{m}^{2}$ i.v. days 1 to 3 $[5,6]$. In the analysis for FLT3-ITD acute myeloid leukemia 22 patients with FLT-ITD positive acute myeloid leukemia had received DAC and 33 patients DA [4]. The complete remission rate was $86 \%$ and $61 \%$ respectively. The four years probability of overall survival was $37 \%$ and $14 \%$ respectively with most prominent overall survival improvement after censoring at the time of allogeneic hematopoietic stem cell transplantation. The four years probability of survival in poor risk patients was $60 \%$ and $18 \%$ respectively.

Although midostaurin is approved in among others the USA and Europe the costs of administration may not be affordable in several countries. In such instance addition of cladribine may offer a cost efficient alternative in FLT3 positive acute myeloid leukemia.

\section{References}

1. Stone RM, Madrklar SJ, Sanford BL, Laumann K, Geyer S, et al. (2017) Midostaurin plus chemotherapy for acute myeloid leukemia with a FLT3 mutation. N Engl J Med 377: 454-464.

2. Zhang QY, Wei XD, Yin QS, Mi RH, Yuan FF, et al. (2017) Sorafenib in combination with chemotherapy as first line therapy for FLT3-ITD positive acute myeloid leukemia. ZhonghuaXue Ye ZaZhi 38(5): 415420.

3. Serve H, Krug U, Wagner R, Sauerland MC, Heinecke A, et al. (2013) Sorafenib in combination with intensive chemotherapy in elderly patients with acute myeloid leukemia: results from a randomized placebo-controlled trial. J Clin Oncol 31(25): 31103118.

4. Libura M, Griebel S, Piatkowska Jakubas B, Pawelczyk M, Florek I, et al. (2016) Cladiribine added to daunorubicine-cytarabine induction prolongs survival of FLT3-ITD positive normal karyotype AML patients. Blood 127(3): 360-362.

5. Holowiecki J, Grosicki S, Robak T, Kyrca Krzemien S, Giebel S, et al. (2004) Addition of cladribine to daunorubicin and cytarabine increase complete remission rate after a single course of inductin treatment in acute myeloid leukemia. Multicenter phase III study. Leukemia 18(5): 989-997.

6. Holowiecki J, Grosicki S, Giebel S, Robak T, KyrczKrzemin S, et al. (2012) Claribine, but not fludarabine added to daunorubicin and cytarabine during inducation prolongs survival of patients with acute myeloid leukemia: a multicheter randomized phase III study. J Clin Oncol 30(20): 2441-2448. 